Pacific Journal of Mathematics

ON THE LIFTING THEORY OF FINITE GROUPS OF LIE TYPE 


\title{
ON THE LIFTING THEORY OF FINITE GROUPS OF LIE TYPE
}

\author{
K. MCGOVERN
}

Let $\mathbf{G}$ be a connected reductive algebraic group defined over a finite field $\mathrm{F}_{q}$ of characteristic $p>0, q=p^{a}$. Let $F$ be a corresponding Frobenius endomorphism such that $\mathbf{G}^{F^{m}}=\left\{g \in G: F^{m}(g)=g\right\}$ is a finite group of Lie type for a positive integer $m$. In this paper we discuss various aspects of the lifting theory of these finite groups.

0. Introduction. The paper is divided into four sections. In $\S 1 . \mathrm{N}$. Kawanaka's norm map is defined and admissible integers are discussed. In $\$ 2$ the lifting theory of $G_{2}(q)$ is described. $\$ 3$ is devoted to liftings of certain principal series representations of groups of adjoint type. Finally, we prove (in \$4) that the duality operation defined by C. W. Curtis [7] commutes with lifting.

We use the notation $\mathbf{G}^{F^{m}}=G\left(q^{m}\right)$, Irr $H=$ set of irreducible characters of a finite group $H, \overline{\mathbf{F}}_{q}=$ algebraic closure of $\mathbf{F}_{q}$, and $A=\left\langle\left. F\right|_{G\left(q^{m}\right)}\right\rangle$. $A$ acts on $G\left(q^{m}\right)$, and is a cyclic group of order $m$. Embed $A$ and $G\left(q^{m}\right)$ into the semidirect product $A \cdot G\left(q^{m}\right)$. If $\chi \in \operatorname{Irr} G\left(q^{m}\right)$ is $F$-invariant, it extends to $\chi^{\prime} \in \operatorname{Irr} A \cdot G\left(q^{m}\right)$. There is a norm map $\Re$ which yields a bijection $\left\{A \cdot G\left(q^{m}\right)\right.$-conjugacy classes in $\left.F \cdot G\left(q^{m}\right)\right\} \leftrightarrow\{$ conjugacy classes of $G(q)\}$ (see $\S 1$ ).

Definition. Let $\theta \in \operatorname{Irr} G(q)$. Then $\psi \in\left(\operatorname{Irr} G\left(q^{m}\right)\right)^{F}$ is the lift of $\theta$ if $\psi$ extends to $\psi^{\prime} \in \operatorname{Irr} A \cdot G\left(q^{m}\right)$ and satisfies $\psi^{\prime}(F y)=C \theta(\Re(y))$ for some constant $C$ and for all $y \in G\left(q^{m}\right)$.

In 1976 a paper of T. Shintani [16] was published which described the lifting theory of the finite groups $\operatorname{GL}(n, q)$. This marked the beginning of the lifting theory of finite groups of Lie type. Kawanaka subsequently developed much of the theory in his papers on $\mathrm{U}(n, q)[11]$ and on $\mathrm{Sp}(2 n, q), \mathrm{SO}(2 n+1, q)$, and $\mathrm{SO}^{ \pm}(2 n, q)$ [12]. We will consider the finite exceptional groups other than $G_{2}(q)$ in future papers (work is in progress).

We wish to thank Professor N. Kawanaka for his constant encouragement and help. We also thank Professors S. Rallis and R. Solomon for many helpful conversations. 
1. The norm map and admissible integers. The material in this section can be found in [12]. Let $\mathbf{G}$ and $F$ be as above. For a finite group $H$ and an element $h \in H$, let $h^{H}$ denote the conjugacy class of $h$ in $H$.

(1.1) LANG'S THEOREM. The mapping $f: g \rightarrow F\left(g^{-1}\right) g$ of $\mathbf{G}$ into $\mathbf{G}$ is surjective.

For $x \in G(q)$, let $A_{x}=Z_{\mathbf{G}}(x) / Z_{\mathbf{G}}(x)^{0} . A_{x}$ is a finite group. For $a \in A_{x}$, choose $y \in f^{-1}\left(r_{a}\right)$, where $r_{a}$ is a representative of $a$ in $Z_{\mathbf{G}}(x)$. Then $y x y^{-1} \in G(q)$, and its $G(q)$-conjugacy class depends only on $x$ and $a$.

(1.2) Definition. Denote the $G(q)$-conjugacy class of $y x y^{-1}$ by $t_{a}(x)$ or $t_{r_{a}}(x)$.

For $y \in G\left(q^{m}\right)$, put $N(y)=y^{F^{m-1}} y^{F^{m-2}} \cdots y^{F} y$ (where $\left.y^{F^{i}}=F^{i}(y)\right)$, and choose $\alpha_{y} \in f^{-1}(y)$. Then $\alpha_{y} N(y) \alpha_{y}^{-1} \in G(q)$, and its $G(q)$-conjugacy class depends only on the class $(F y)^{A \cdot G\left(q^{m}\right)}$.

(1.3) Definition. Let $n_{\mathbf{G}}:\left\{(F y)^{A \cdot G\left(q^{m}\right)}: y \in G\left(q^{m}\right)\right\} \rightarrow\left\{x^{G(q)}: x \in\right.$ $G(q)\}$ be defined by

$$
n_{\mathbf{G}}\left((F y)^{A \cdot G\left(q^{m}\right)}\right)=\left(\alpha_{y} N(y) \alpha_{y}^{-1}\right)^{G(q)}
$$

for $y \in G\left(q^{m}\right)$. Then $n_{\mathbf{G}}$ is bijective.

(1.4) Definition. For $x \in G(q)$, let $\bar{x}=$ image of $x$ in $A_{x}$. Then a positive integer $l$ is admissible for $\mathbf{G}$ and $F$ if $(\operatorname{ord}(x), l)=1$ for all $x \in G(q)$, where ord $(\bar{x})=$ order of $\bar{x}$ in $A_{x}$. (It is known (see [18]) that the splitting of the class of $x \in \mathbf{G} \cap G\left(q^{m}\right)$ into classes in $G\left(q^{m}\right)$ is in 1-1 correspondence with the elements of $H^{1}\left(F^{m}, Z_{\mathbf{G}}(x) / Z_{\mathbf{G}}(x)^{0}\right)$ for any $m$. Thus the consideration of admissible integers is a natural one for our purposes.)

(*) Unless stated otherwise, we assume $m$ is admissible. Thus, there is a unique element $x(m)$ of $\langle\bar{x}\rangle$ such that $x(m)^{m}=\bar{x}$.

(1.5) Definition. The map $t_{\mathbf{G}}: \dot{G}(q) \rightarrow\left\{x^{G(q)}: x \in G(q)\right\}$ is given by

$$
t_{\mathbf{G}}(x)=t_{x(m)}(x)
$$

for $x \in G(q)$. 
(1.6) REMARKS.

(i) $t_{\mathbf{G}}$ induces a permutation of the set of conjugacy classes of $G(q)$.

(ii) $t_{\mathbf{G}}$ is the identity map in case $Z_{\mathbf{G}}(x)=Z_{\mathbf{G}}(x)^{0}$ for all $x \in G(q)$.

(iii) $t_{\mathbf{G}}(x)=x$ in case $x \in Z_{\mathbf{G}}(x)^{0}$.

(1.7) Definition. Let $\Re_{\mathbf{G}}=t_{\mathbf{G}}^{-1} \circ n_{\mathbf{G}}$ and $\Re_{\mathbf{G}}=\Re_{\mathbf{G}}^{-1}$.

(1.8) THEOREM.

(i)

$$
\left\{x^{G(q)}: x \in G(q)\right\} \underset{\boldsymbol{\pi}_{\mathbf{G}}}{\stackrel{\Re_{\mathbf{G}}}{\rightleftarrows}}\left\{(F y)^{A \cdot G\left(q^{m}\right)}: y \in G\left(q^{m}\right)\right\}
$$

are bijections.

(ii) For any $y \in G\left(q^{m}\right)$,

$$
\left|(F y)^{A \cdot G\left(q^{m}\right)}\right|\left|G\left(q^{m}\right)\right|^{-1}=\left|\Re_{\mathbf{G}}\left((F y)^{A \cdot G\left(q^{m}\right)}\right)\right||G(q)|^{-1} .
$$

(iii) For $y \in G\left(q^{m}\right)$, if $Z_{\mathbf{G}}(N(y))^{0} \ni N(y)\left(=(F y)^{m}\right)$, then

$$
\Re_{\mathbf{G}}\left((F y)^{A \cdot G\left(q^{m}\right)}\right)=n_{\mathbf{G}}\left((F y)^{A \cdot G\left(q^{m}\right)}\right) .
$$

In particular, this is the case if $N(y)$ is semisimple or $\mathbf{G}=\mathrm{GL}\left(n, \overline{\mathbf{F}}_{q}\right)$.

(iv) Let $\mathbf{T}$ be an $F$-stable torus in $G$, and let $T=\mathbf{T}^{F^{m}}$. For $t \in T$ we have

$$
\Re_{\mathbf{G}}\left((F t)^{A \cdot G\left(q^{m}\right)}\right)=N(t)^{G(q)}
$$

(v) Let $a \in G(q)$ such that $(\operatorname{ord}(a), m)=1$. Then

$$
\Re_{\mathbf{G}}\left((F a)^{A \cdot G\left(q^{m}\right)}\right)=\left(a^{m}\right)^{G(q)} .
$$

(1.9) Let $\mathbf{H}$ be an $F$-stable connected algebraic subgroup of $\mathbf{G}$ for which $m$ is admissible. Put $H\left(q^{m}\right)=\mathbf{H}^{F^{m}}$. Then $\Re_{\mathbf{H}}\left(h^{H(q)}\right) \subset \Re_{\mathbf{G}}\left(h^{G(q)}\right)$ for any $h \in H(q)$.

We will abbreviate $\Re_{\mathbf{G}}$ as $\Re$, and $\Re_{\mathbf{G}}$ as $\Re$.

Definitions (1.2)-(1.5) and (1.7) are due to Kawanaka.

2. The lifting theory of $G_{2}(q)$. The following theorem holds for $G_{2}(q), q=p^{a}$, for sufficiently large $p$, depending on the rank of $\mathbf{G}_{2}$ (see [9], [13]), and for $p=2,3$. 
(2.1) TheOREM. Let $m$ be a positive integer which satisfies $(m, 2)=$ $(m, 3)=1$. Let $\Re$ be the norm map defined in (1.7). Then

(i) For any irreducible character $\chi$ of $G_{2}(q)$, there is a unique $\psi_{\chi} \in$ $\left(\operatorname{Irr} G_{2}\left(q^{m}\right)\right)^{F}$ such that

$$
\chi(\Re(y))=\varepsilon \delta \psi_{\chi}^{\prime}(F y)
$$

for all $y \in G_{2}\left(q^{m}\right)$, where $\varepsilon= \pm 1, \delta$ is an $m$ th root of 1 (both independent of $y$ ), and $\psi_{\chi}^{\prime}$ is an extension of $\psi_{\chi}$ to $A \cdot G_{2}\left(q^{m}\right)$.

(ii) The map $\chi \rightarrow \psi_{\chi}$ gives a bijection between $\operatorname{Irr} G_{2}(q)$ and $\left(\operatorname{Irr} G_{2}\left(q^{m}\right)\right)^{F}$.

Proof. Notation will be as in [5], [6], [12]. We first identify the admissible integers for $G_{2}(q)$.

(2.2) LEMMA. The admissible integers for $G_{2}(q)$ are those integers $m$ satisfying $(m, 2)=(m, 3)=1$.

Proof of Lemma (2.2). Assume $m$ is admissible. We consider the cases $p \neq 2,3, p=2$, and $p=3$ separately.

(i) $p \neq 2$, 3. If $q \equiv 1(\bmod 3)$, consider the element $x=h(\omega, \omega, \omega) y$, where $y$ is a regular unipotent element of $\operatorname{SL}(3, q)$. Then $x$ has order $3 p$, and $A_{x}=Z_{\mathbf{G}_{2}}(x) / Z_{\mathbf{G}_{2}}(x)^{0}$ is isomorphic to $\mathbf{Z}_{3}$, a cyclic group of order 3 . Furthermore $\bar{x}$ has order 3 in $A_{x}$. A similar analysis for $q \equiv-1(\bmod 3)$, together with the information above, yields $(m, 3)=1$. To see that we must have $(m, 2)=1$, consider $x=h(-1,-1,1) x_{b}(1) x_{c}(1)$ if $q \equiv 1$ $(\bmod 3)\left(\right.$ or $x=h(-1,-1,1) x_{b}(1) x_{c}(\lambda)$ if $\left.q \equiv-1(\bmod 3)\right)$. Then $A_{x}$ is isomorphic to a cyclic group of order 2 , and $\bar{x}$ has order 2 in $A_{x}$.

(ii) $p=2$. As above we must have $(m, 3)=1$. Since 2 is a bad prime for $\mathbf{G}_{2}, Z_{\mathbf{G}_{2}}(x) / Z_{\mathbf{G}_{2}}(x)^{0} \cong\langle x\rangle \cong \mathbf{Z}_{2}$, a cyclic group of order 2 , for a regular unipotent element $x$. This yields $(m, 2)=1$.

(iii) $p=3$. Arguments are the same as in (ii), with the roles of 2 and 3 interchanged.

\section{(2.2) REMARKS.}

(i) Since $\mathbf{G}_{2}$ is simply connected (as an algebraic group), the centralizers of semisimple elements are connected. These elements, then, will not impose restrictions on $m$.

(ii) For $p \neq 2,3$ all unipotent elements $u$ are contained in $Z_{\mathbf{G}_{2}}(u)^{0}$. Hence these too impose no restrictions on $m$. In any case, $\left|Z_{\mathbf{G}_{2}}(u) / Z_{\mathbf{G}_{2}}(u)^{0}\right|$ is divisible only by the primes 2 and 3 . 
(iii) Examination of the conjugacy classes of $G_{2}(q)$ ([5], [6]) shows that it is sufficient that $(m, 2)=(m, 3)=1$ for an integer $m$ to be admissible. With this observation, the proof of Lemma (2.2) is complete.

Now the remainder of the proof proceeds much as that in [12]. We use a version of Brauer's characterization of characters (see Lemma (1.5) of [11]). After invoking (1.8) (i) and (ii), we see that it is sufficient to prove the following lemma:

(2.3) LEMMA. Let $E=\langle g\rangle \times S$ be an elementary subgroup of $G_{2}(q)$. (So $g \in G_{2}(q)$ and $S$ is an s-group for some prime number $s$ satisfying $(\operatorname{ord}(g), s)=1$.) Then there exists an $F$-stable subgroup $\mathbf{H} \subseteq G_{2}$ with $H\left(q^{m}\right)$ contained in $G_{2}\left(q^{m}\right)$ and a bijection

$$
\mathfrak{R}_{H\left(q^{m}\right)}=\left\{x^{H(q)}: x \in H(q)\right\} \rightarrow\left\{(F y)^{A \cdot H\left(q^{m}\right)}: y \in H\left(q^{m}\right)\right\}
$$

such that

(i) $E \subset H\left(q^{m}\right)$,

(ii) $\Re_{H\left(q^{m}\right)}\left(x^{H(q)}\right) \subset \mathfrak{R}\left(x^{G_{2}(q)}\right)$ for any $x \in H(q)$,

(iii) For any irreducible character $\phi$ of $A \cdot H\left(q^{m}\right), \phi \circ \mathfrak{N}_{H\left(q^{m}\right)}$ is either zero or an irreducible character of $H(q)$ up to a constant multiple in $\mathbf{Z}\left(e^{2 \pi i / m}\right)$. (Note that for any class function $\eta$ on $A \cdot G_{2}\left(q^{m}\right)$, $\eta \circ \mathfrak{T}$ is the class function on $G(q)$ defined by $\eta \circ \Re(x)=\eta\left(m_{x}\right)$ for $x \in G(q)$ and $m_{x} \in\left(x^{G_{2}(q)}\right)$.)

Proof of Lemma (2.3). First assume $p \neq 2$, 3. Let $E=\langle g\rangle \times S$ be an elementary subgroup of $G_{2}(q)$. Let $g=g_{s} g_{u}$ be the Jordan decomposition of $g$, where $g_{s}$ is semisimple and $g_{u}$ is unipotent. Since the center of $\mathbf{G}_{2}$ is trivial, we have $Z_{\mathbf{G}_{2}}(x) \varsubsetneqq G_{2}$ for all $x \in \mathbf{G}_{2}$. In many cases, then, we appeal to the lifting theory of $Z_{\mathbf{G}_{2}}(x)$. The following cases will be considered:

(1) $g_{s} \neq 1$,

(2) $g=g_{u}, s=2$,

(3) $g=g_{u}, s=3$,

(4) $g=g_{u}, s=p$,

(5) $g=g_{u}, s \neq 2,3, p$.

Case 1. If $g_{s} \neq 1$, then $1 \neq Z_{\mathbf{G}_{2}}\left(g_{s}\right)=Z_{\mathbf{G}_{2}}\left(g_{s}\right)^{0}$. In view of the results in [3], [5], [6], [10] it suffices to prove (ii) and (iii) above for $\mathbf{H}=\operatorname{SL}\left(2, \overline{\mathbf{F}}_{q}\right)$, $\operatorname{SL}\left(3, \overline{\mathbf{F}}_{q}\right), \operatorname{GL}\left(2, \overline{\mathbf{F}}_{q}\right)$, and $F$-stable maximal tori $\mathbf{T} \leq \mathbf{G}_{2}$. (Then $\mathbf{H}^{F}=$ $\mathrm{SL}(2, q), \operatorname{SL}(3, q), \operatorname{SU}(3, q), \mathrm{GL}(2, q), \mathrm{U}(2, q)$.) It is easy to see that all odd integers are admissible for $\operatorname{SL}\left(2, \overline{\mathbf{F}}_{q}\right)$ and that all integers relatively 
prime to 3 are admissible for $\operatorname{SL}\left(3, \overline{\mathbf{F}}_{q}\right)$. It is also straightforward that irreducible characters of $\operatorname{SL}(2, q)$ (respectively $\operatorname{SL}(3, q)$ ) always lift to $F$-invariant irreducibles of $\operatorname{SL}\left(2, q^{m}\right)$ (respectively $\operatorname{SL}\left(3, q^{m}\right)$ ) if and only if $m$ is admissible, for $q$ odd. (If $q=2^{a}$, irreducibles of $\operatorname{SL}(2, q)$ always lift.) All integers are admissible for $\mathrm{GL}\left(2, \overline{\mathbf{F}}_{q}\right)$ and $F$-stable maximal tori $\mathbf{T}$, and the lifting theory for these groups is known. If $\operatorname{ord}\left(g_{s}\right) \neq 2,3$, $H=Z_{\mathbf{G}_{2}}\left(g_{s}\right)^{0}, H\left(q^{m}\right)=\mathbf{H}^{F^{m}}$, and $\Re_{H\left(q^{m}\right)}=\Re_{\mathbf{H}}$ satisfy conditions (i)(iii) above. In case ord $\left(g_{s}\right)=2$, we may use $\mathbf{H}=\operatorname{SL}\left(2, \overline{\mathbf{F}}_{q}\right) \circ \operatorname{SL}\left(2, \overline{\mathbf{F}}_{q}\right)$ (central product) instead of the full centralizer of $g_{s}$, and (i)-(iii) are satisfied with $H\left(q^{m}\right)=\operatorname{SL}\left(2, q^{m}\right) \circ \operatorname{SL}\left(2, q^{m}\right)$ and $\Re_{H\left(q^{m}\right)}=\Re_{\mathbf{H}}$. In case $g_{s}$ is an element of order 3 whose centralizer has order $q(q \pm 1)(q \mp 1)^{2}$, an appropriate subgroup of $Z_{\mathbf{G}_{2}}\left(g_{s}\right)$ may be used for $\mathbf{H}$ in a similar manner.

In the remaining cases, $g=g_{u}$ and, since $g_{u} \in Z_{\mathbf{G}_{2}}\left(g_{u}\right)^{0}$, the mapping $t_{G_{2}}$ is trivial on $g_{u}$.

Case 2. $S$ nontrivial $\Rightarrow$ there is an element $1 \neq x \in Z(S)$ (the center of $S$ ). Then $\left\langle g_{u}\right\rangle \times S \leq Z_{\mathbf{G}_{2}}(x)$ and this is considered in case 1 .

Case 3 and Case 5 are proved similarly.

Case 4. This can only occur if $E=\left\langle g_{u}\right\rangle$ or $E=S$, where $S$ is a $p$-group, since (ord $\left.g_{u}, s\right)=1$. If $E=\left\langle g_{u}\right\rangle$, we invoke Gyoja's result on the lifting theory of exponential unipotent subgroups, and the case $E=S$ follows similarly. (This is where the constraint on $p$ is needed.)

This completes the proof of Lemma 2.3, and thus completes the proof of Theorem (2.1) in case $p \neq 2,3$.

If $p=2$ or 3 , the lemma, hence the theorem, is proved essentially as above, except that now regular unipotent elements $u \notin Z_{\mathbf{G}_{2}}(u)^{0}$. It is necessary then to reconsider the case $g=g_{u}, s=2$ (if $p=3$ ), and $g=g_{u}$, $s=3$ (if $p=2$ ) in this context. Letting $\mathbf{H}=\left\langle g_{u}\right\rangle \times S$ and $\mathfrak{K}_{H\left(q^{m}\right)}^{-1}(F x y)$ $=\left(x^{m} y^{m}\right)^{H(q)}$ for $x \in\left\langle g_{u}\right\rangle$ and $y \in S$ proves the lemma in case $p=2$ or 3 , and the theorem is established.

Theorem (2.1) holds for all $p$, in case we require that $(m, p)=1$. One would use Theorem (1.8)(v) instead of [9, Prop. 4.4] in the proof above.

3. Principal series representations. Let $\mathbf{G}$ be a simple adjoint algebraic group, and let $F$ be a Frobenius endomorphism such that $\mathbf{G}^{F^{n}}=$ $G\left(q^{n}\right)$ is a finite untwisted group of adjoint type. Let $(W, R)$ be the Coxeter system of $G$, and for $J \subseteq R$, denote by $W_{J}$ the parabolic subgroup of $W$ corresponding to $J$. Fix a positive integer $m$ and assume lifting from 
$\operatorname{Irr} G(q)$ to $\operatorname{Irr} G\left(q^{m}\right)$ occurs for all $\phi \in \operatorname{Irr} G(q)$, and similarly for all parabolics of $G(q)$.

Let $T(q)$ be the standards torus of $G(q)$. For any $\lambda \in \operatorname{Irr} T(q)$, $W(\lambda)=\left\{w \in W: \lambda^{w}=\lambda\right\}$ is a reflection group with fundamental system $S$, and $(W(\lambda), S)$ is a Coxeter system. See [14] for details. For each $J \subseteq R, W(\lambda) \cap W_{J}$ is a parabolic subgroup of $W(\lambda)$. (See [15].) Fix $\lambda \in \operatorname{Irr} T(q)$ so $W(\lambda)$ satisfies the following: as $J$ ranges over all subsets of $R, J \cap S$ ranges over all subsets of $S$. Then $\left\{W(\lambda) \cap W_{J}: J \subseteq R\right\}$ consists of all parabolic subgroups of $W(\lambda)$. (This occurs, for example, when $W(\lambda)$ is a parabolic subgroup of $W$.)

Composing $\lambda$ with $N_{m}$ (the usual map on tori), it is evident that $\lambda \circ N_{m} \in \operatorname{Irr} T\left(q^{m}\right)$, and $W(\lambda)=W\left(\lambda \circ N_{m}\right)$. The constituents of $\left(\lambda \circ N_{m}\right)_{B\left(q^{m}\right)}^{G\left(q^{m}\right)}$ are parametrized by the irreducible characters of $W(\lambda)$, as are the constituents of $\lambda_{B(q)}^{G(q)}$. We denote by $\zeta_{\psi, m}$ (respectively $\zeta_{\psi}$ ) the unique constituent of $\left(\lambda \circ N_{m}\right)_{B\left(q^{m}\right)}^{G\left(q^{m}\right)}$ (respectively $\left.\lambda_{B(q)}^{G(q)}\right)$ corresponding to $\psi \in \operatorname{Irr} W(\lambda)$. If $W(\lambda)$ is of type $G_{2}, E_{7}$, or $E_{8}$, we consider only those irreducible characters $\psi$ of $W(\lambda)$ which are uniquely determined by the multiplicities $\left\{\left(\psi, 1_{W_{J} \cap W(\lambda)}^{W(\lambda)}\right): J \subseteq R\right\}$. This occurs for almost all the irreducibles (see [4]).

Let $A(\lambda)$ be the generic algebra associated with $(W(\lambda), S)$. Then $A(\lambda)$ is an associative $C[t]$-algebra with generators $\left\{a_{w}: w \in W(\lambda)\right\}$ and satisfies:

(i) $a_{w} a_{w_{i}}=a_{w w_{t}}, l\left(w w_{i}\right)=l(w)+1$,

(ii) $a_{w} a_{w_{l}}=t a_{w w_{l}}+(t-1) a_{w}, l\left(w w_{i}\right)=l(w)-1$, for $w \in W(\lambda), w_{i} \in S$.

For a subset $J \subseteq R, A_{J}(\lambda)$ is a subalgebra of $A(\lambda)$ with generators $\left\{a_{w}: w \in W_{J} \cap W(\lambda)\right\}$ since $W_{J} \cap W(\lambda)$ is a parabolic subgroup of $W(\lambda)$. Let $\mathcal{H}\left(G\left(q^{m}\right), B\left(q^{m}\right), \lambda \circ N_{m}\right)$ be the Hecke algebra of $G\left(q^{m}\right)$ corresponding to $\lambda \circ N_{m}$. There are isomorphisms $f_{m}: t \rightarrow q^{m}$ and $f_{0}: t \rightarrow 1$ with extensions $f_{m}^{*}$ and $f_{0}^{*}$ such that the specialized algebra $A(\lambda)_{f_{m}^{*}} \cong$ $\mathcal{H}\left(G\left(q^{m}\right), B\left(q^{m}\right), \lambda \circ N_{m}\right)=\mathcal{H}\left(\lambda \circ N_{m}\right)$ and $A(\lambda)_{f_{0}^{*}} \cong W(\lambda)$. Then $\left\{a_{w, f_{m}^{*}}\right\}$ forms a basis for $\mathcal{H}\left(\lambda \circ N_{m}\right) ;\left\{a_{w, f_{0}^{*}}\right\}=W(\lambda)$. For any irreducible character $\chi$ of $A(\lambda)_{K}(K=$ splitting field for $A(\lambda)), \chi_{f_{0}^{*}} \in \operatorname{Irr} W(\lambda)$ and $\chi_{f_{m}^{*}} \in \operatorname{Irr} \mathcal{H}\left(\lambda \circ N_{m}\right)$. We sometimes say $\zeta_{\psi, m}=\chi_{f_{m}^{*}}$, though actually $\left.\zeta_{\psi, m}\right|_{\mathcal{S C}_{\left(\lambda \circ N_{m}\right)}}=\chi_{f_{m}^{*}}$

Since $F$ acts trivially on $W$, each $\zeta_{\psi, m}$ may be regarded as an irreducible character of $A \cdot G\left(q^{m}\right)$. Call the extended character $\tilde{\zeta}_{\psi, m}$. From Shintani's work [16] and Lemma 1.1.9 in [2] we see that $\left(\lambda \circ N_{m}\right)_{B\left(q^{m}\right)}^{G\left(q^{m}\right)}(F y)$ $=c \lambda_{B(q)}^{G(q)}(\Re(y))$ for all $y \in G\left(q^{m}\right)$ and some constant $c$, where 
$\left(\lambda \circ N_{m}\right)_{B\left(q^{m}\right)}^{G\left(q^{m}\right)}$ is regarded as a character of $A \cdot G\left(q^{m}\right)$ and $\Re$ is the norm map in [2]. In [2, §2], T. Asai characterized the lifting theory of constituents of $\mathrm{I}_{B(q)}^{G(q)}$, where $G(q)$ is $\mathrm{GL}(n, q), \mathrm{U}(n, q), \operatorname{Sp}(2 n, q)$, $\mathrm{SO}(2 n+1, q)$, or $\mathrm{SO}^{ \pm}(n, q)$. Because of the nature of the decomposition of $\left(\lambda \circ N_{m}\right)_{B\left(q^{m}\right)}^{G\left(q^{m}\right)}$ and $\lambda_{B(q)}^{G(q)}$, the general ideas in [2, §2] also apply to our setting. In particular, for $\chi^{\prime} \in \operatorname{Irr}\left(W(\lambda) \cap W_{J}\right)$, let $\chi^{\prime W(\lambda)}$ $=\Sigma_{\phi} n_{\chi^{\prime}, \phi} \phi$, as $\phi$ varies over $\operatorname{Irr} W(\lambda)$, for nonnegative integers $n_{\chi^{\prime}, \phi}$. Then $\zeta_{\chi^{\prime}, m}^{G\left(q^{m}\right)}=\Sigma_{\phi} n_{\chi^{\prime}, \phi} \zeta_{\phi, m}$ and $\tilde{\zeta}_{\chi^{\prime}, m}^{A \cdot G\left(q^{m}\right)}=\Sigma_{\phi} n_{\chi^{\prime}, \phi} \tilde{\zeta}_{\phi, m}$, where $\left.\zeta_{\chi^{\prime}, m}\right|_{A_{J}(\lambda)_{f m}}$ $=\chi_{f_{m}^{*}}$, for $\chi \in \operatorname{Irr} A_{J}(\lambda)_{K}$ and $\tilde{\zeta}_{\chi^{\prime}, m}$ is the extension of $\zeta_{\chi^{\prime}, m}$ to $A \cdot P_{J}\left(q^{m}\right)$.

(3.1) THEOREM. Notation as above. For $\zeta_{\psi} \in \lambda_{B(q)}^{G(q)}$ and $\psi \in \operatorname{Irr} W(\lambda)$, one has $\operatorname{lift}\left(\zeta_{\psi}\right)=\zeta_{\psi, m}$.

Proof. We use induction on the rank of $(W(\lambda), S)$. For rank $(W(\lambda), S)=1, \lambda_{B(q)}^{G(q)}=\mathrm{St}_{G(q), \lambda}+\zeta(\lambda)$, where $\mathrm{St}_{G(q), \lambda}$ is the generalized Steinberg character and $\zeta(\lambda)$ is the generalized identity in $\operatorname{Irr} G(q)$ corresponding to $\lambda$. Then Gyoja's work implies that $\mathrm{St}_{G(q), \lambda}$ lifts to $\mathrm{St}_{G\left(q^{m}\right), \lambda} \circ N_{m}$ and $\zeta(\lambda)$ lifts to $\zeta\left(\lambda \circ N_{m}\right)$ (see $\S 6$ of [9], and [14]).

Now assume $\operatorname{rank}(W(\lambda), S) \geq 2$, and the theorem holds for all proper parabolics $P_{J}(q)$ of $G(q)$. Since $\left(\lambda \circ N_{m}\right)_{B\left(q^{m}\right)}^{G\left(q^{m}\right)}(F y)=c \lambda_{B(q)}^{G(q)}(\mathcal{T}(y))$, it follows that $\operatorname{lift}\left(\zeta_{\psi}\right)=\zeta_{\phi}$ for some $\phi \in \operatorname{Irr} W(\lambda)$, by Lemma 1.1.8 of [2]. Restricting $\zeta_{\psi}$ and $\zeta_{\phi}$ to the appropriate parabolic subgroups gives $\left.\psi\right|_{W_{J} \cap W(\lambda)}=\left.\phi\right|_{W_{J} \cap W(\lambda)}$ (as $J$ ranges over all subsets of $R$ ), using the induction hypothesis.

Then since $\left\{W_{J} \cap W(\lambda): J \subseteq R\right\}$ is the set of all parabolic subgroups of $W(\lambda)$, the proof of the theorem is completed by using a theorem of Benson and Curtis [4]:

Let $(W, S)$ be an irreducible Coxeter system of rank $\geq 2$. Let $\chi_{1}$, $\chi_{2} \in \operatorname{Irr} W$ (with exceptions in types $G_{2}, E_{7}$, and $E_{8}$ as noted above). Then $\left.\chi_{1}\right|_{W_{J}}=\left.\chi_{2}\right|_{W_{J}}$ for all $J \subset S$ implies $\chi_{1}=\chi_{2}$.

4. Duality and lifting. Let $G$ be a finite group of Lie type, and $(W, R)$ the Coxeter system of $G$. C. W. Curtis has defined two operations in $\operatorname{char}_{\mathbf{Z}}(G)$, the ring of complex valued characters of $G$ [7].

(1.4) Definition. Let $\zeta$ be a character of $G$ and let $M$ be the module affording $\zeta$. For any subset $J \subseteq R$, let $\zeta_{\left(P_{J}\right)}$ be the character of $P_{J}$ afforded by $\operatorname{inv}_{V_{J}}(M)=\left\{m \in M: v m=m \forall v \in V_{J}\right\}$. 
(4.2) Definition. Let $G,(W, R), \zeta$ be as above. The dual $\zeta^{*}$ of $\zeta$ is defined to be

$$
\zeta^{*}=\sum_{J \subseteq R}(-1)^{\mu /} \zeta_{\left(P_{J}\right)}^{G}
$$

Extending the definition of $\zeta_{\left(P_{J}\right)}$ by additivity, there are well-defined maps $\operatorname{char}_{\mathbf{Z}}(G) \rightarrow \operatorname{char}_{\mathbf{z}}\left(P_{J}\right)$ via $\zeta \rightarrow \zeta_{\left(P_{J}\right)}$ and $\operatorname{char}_{\mathbf{z}}(G) \rightarrow \operatorname{char}_{\mathbf{z}}(G)$ via $\zeta \rightarrow \zeta^{*}$. The duality operation is a generalization of the construction of the Steinberg character $\mathrm{St}_{G}$. In fact, $1_{G}^{*}=\mathrm{St}_{G}$. D. Alvis has proved that $\pm \zeta^{*} \in \operatorname{Irr} G$ if $\zeta \in \operatorname{Irr} G$, and that $\zeta^{* *}=\zeta[1]$.

(4.3) Theorem. Suppose $\zeta \in \operatorname{Irr} G(q)$ lifts to $\psi \in \operatorname{Irr} G\left(q^{m}\right)$. Then $\varepsilon_{\zeta} \zeta^{*} \in \operatorname{Irr} G(q)$ also lifts, and $\operatorname{lift}\left(\varepsilon_{\zeta} \zeta^{*}\right)=\varepsilon_{\psi} \psi^{*} \in \operatorname{Irr} G\left(q^{m}\right)$, for constants $\varepsilon_{\zeta}, \varepsilon_{\psi}$. The converse is also true, since ${ }^{*}$ is an involution.

Proof. For a character $\theta$ of $G, \theta^{*}=\sum_{\tilde{\phi}^{\subseteq} \subseteq R}(-1)^{\mu^{\prime}} \theta_{\left(P_{J}\right)}^{G}$, where $\theta_{\left(P_{J}\right)}=$ $\sum a_{i} \tilde{\phi}_{l}$, summed over all $\phi_{l} \in \operatorname{Irr} L_{J}$, and $\tilde{\phi}_{i}$ is the extension of $\phi_{i}$ to $P_{J}$ obtained by putting $V_{J}$ in its kernel.

Assume $\zeta$ lifts to $\psi_{\zeta}$. We must show (i) $\varepsilon_{\psi} \psi^{*}$ is $F$-invariant and (ii) $\left(\varepsilon_{\psi} \psi^{*}\right)^{\prime}(F y)=\varepsilon_{\zeta} \zeta^{*}(\mathcal{T}(y))$ for all $y \in G\left(q^{m}\right)$ (where $\left(\varepsilon_{\psi} \psi^{*}\right)^{\prime}$ is an extension of $\varepsilon_{\psi} \psi^{*}$ to $\left.A \cdot G\left(q^{m}\right)\right)$. That $\varepsilon_{\psi} \psi^{*}$ if $F$-invariant follows from the $F$-invariance of $\psi$ and from the properties of $\psi^{*}$.

The original proof of (ii) is given now, and holds only in case $F$ is of untwisted type. We first show that $\zeta_{\left(P_{J}(q)\right)}(\Re(l v))=\psi_{\left(A \cdot P_{J}\left(q^{m}\right)\right)}^{\prime}(F l v)$ for $l \in L_{J}\left(q^{m}\right)$ and $v \in V_{J}\left(q^{m}\right)$. By definition of the norm map $\mathcal{O}$ we have $\Re(l v)=\mathscr{T}(l) v^{\prime}$ for some $v^{\prime} \in V_{J}(q)$ since $V_{J}\left(q^{m}\right)$ is normalized by $L_{J}\left(q^{m}\right)$. Thus

$$
\zeta_{\left(P_{J}(q)\right)}(\Re(l v))=\zeta_{\left(P_{J}(q)\right)}\left(\Re(l) v^{\prime}\right)=\left.\zeta\right|_{L_{J}(q)}(\Re(l)) .
$$

Now we may assume

$$
\psi_{\left(A \cdot P_{J}\left(q^{m}\right)\right)}^{\prime}(F l)=\left.\psi^{\prime}\right|_{A \cdot L_{J}\left(q^{m}\right)}(F l)
$$

Since lift $\zeta=\psi$, it follows that

$$
\left.\varepsilon_{\psi} \psi^{\prime}\right|_{A \cdot L_{J}\left(q^{m}\right)}(F l)=\left.\zeta\right|_{L_{J}(q)}(\Re(l))
$$

and hence

$$
\zeta_{\left(P_{J}(q)\right)}(\Re(l v))=\varepsilon_{\psi} \psi_{\left(A \cdot P_{J}\left(q^{m}\right)\right)}^{\prime}(F l v)
$$


Now we use Lemma 1.1.9 in [2] to finish the proof. Combining it with the above, we have

$$
\varepsilon_{\psi} \psi_{\left(A \cdot P_{J}\left(q^{m}\right)\right)}^{\prime A \cdot G\left(q^{m}\right)}(F y)=\zeta_{\left(P_{J}(q)\right)}^{G(q)}(\Re(y)) \text { for all } y \in G\left(q^{m}\right)
$$

Thus

$$
\begin{aligned}
\left(\varepsilon_{\zeta} \zeta^{*}\right)(\Re(y)) & =\sum_{J \subseteq R}(-1)^{|J|} \varepsilon_{\zeta} \zeta_{\left(P_{J}(q)\right)}^{G(q)}(\Re(y)) \\
& =\sum_{J \subseteq R}(-1)^{|J|} \varepsilon_{\psi} \psi_{\left(A \cdot P_{J}\left(q^{m}\right)\right)}^{\prime A \cdot G\left(q^{m}\right)}(F y),
\end{aligned}
$$

and the proof is complete.

We wish to heartily thank Professor Kawanaka for providing us with the following explicit proof. In (II), below, he generalizes the theorem to include the case of $F$ acting nontrivially on $P_{J}\left(q^{m}\right)$.

(I) If $F$ fixes $P_{J}\left(q^{m}\right)$, then

$$
\begin{aligned}
\zeta_{\left(P_{J}(q)\right)}(\Re(l v)) & =\left|V_{J}(q)\right|^{-1} \sum_{v^{\prime}} \zeta\left(\Re(l v) v^{\prime}\right) \quad\left(v^{\prime} \in V_{J}(q)\right) \\
& =\left|V_{J}(q)\right|^{-1} \sum_{v^{\prime}} \zeta\left(\Re(v l) v^{\prime}\right) \\
& =\left|V_{J}(q)\right|^{-1}\left(\frac{\left|V_{J}(q)\right|}{\left|V_{J}\left(q^{m}\right)\right|} \sum_{v} \zeta(\Re(l v))\right) \quad\left(v \in V_{J}\left(q^{m}\right)\right) \\
& =\left|V_{J}\left(q^{m}\right)\right|^{-1} \sum_{v} \varepsilon_{\psi} \psi^{\prime}(F l v)=\chi_{J}(F l v),
\end{aligned}
$$

where $\chi_{J}$ is a character of $A \cdot P_{J}\left(q^{m}\right)$ afforded by $\operatorname{inv}_{V_{J}\left(q^{m}\right)}\left(M_{\psi^{\prime}}\right)$ (and $M_{\psi^{\prime}}$ affords $\psi^{\prime}$ ). Then

$$
\zeta^{*}(\Re(y))=\sum_{J \subseteq R}(-1)^{\mu /} \zeta_{\left(P_{J}(q)\right)}^{G(q)}(\Re(y))=\sum_{J \subseteq R}(-1)^{\mid \jmath} \chi_{J}^{A \cdot G\left(q^{m}\right)}(F y)
$$

(by using Lemma 1.1.9 on [2]).

But this is an extension of $\psi^{*}$ to $A \cdot G\left(q^{m}\right)$, and we may conclude that $\left(\varepsilon_{\zeta} \zeta^{*}\right)(\mathcal{R}(y))=\varepsilon_{\psi} \psi^{*}(F y)$.

(II) If $F$ acts nontrivially on $P_{J}\left(q^{m}\right)$, let $\left\{P_{J}\left(q^{m}\right)=P_{J_{0}}\left(q^{m}\right)\right.$, $\left.P_{J_{1}}\left(q^{m}\right), \ldots, P_{J_{n}}\left(q^{m}\right)\right\}$ be the $F$-orbit of $P_{J}\left(q^{m}\right)$. Let $\chi_{J}$ be the character of $A \cdot G\left(q^{m}\right)$ satisfying

$$
\left.\chi_{J}\right|_{G\left(q^{m}\right)}=\sum_{i=0}^{n}\left(\left(\zeta \circ F^{i}\right)_{\left(P_{J_{i}}\left(q^{m}\right)\right)}\right)^{G\left(q^{m}\right)}
$$


and $F$ permutes the $(n+1)$ characters which are summands of $\left.\chi_{J}\right|_{G\left(q^{m}\right)}$. Then $\chi_{J}(F x)=0$ for all $x \in G\left(q^{m}\right)$, and the result follows by summing over all $J \subseteq R$ as above.

(4.4) CoRollaRY. Let $\lambda \in \operatorname{Irr} T(q)$ and $\zeta_{\phi} \in \lambda_{B(q)}^{G(q)}$ (notation as in §3). Then $\zeta_{\phi}$ lifts to $\zeta_{\phi, m} \in\left(\lambda \circ N_{m}\right)_{B\left(q^{m}\right)}^{G\left(q^{m}\right)}$ if and only if $\zeta_{\varepsilon_{W(\lambda)} \cdot \phi}$ lifts to $\zeta_{\varepsilon_{\mid W(\lambda)} \cdot \phi, m}$.

Proof. In [15] it is proved that $\zeta_{\phi}^{*}=\zeta_{\varepsilon_{W(\lambda)} \cdot \phi^{*}}$

(4.5) COROLlaRY. The Steinberg character $\mathrm{St}_{G}$ always lifts.

Proof. $1_{G}^{*}=\mathrm{St}_{G}$.

Corollary (4.5) has been proved independently by Gyoja [9, Lemma $6.2(3)]$, in case the algebraic group giving rise to $G(q), G\left(q^{m}\right)$ has a connected center.

\section{REFERENCES}

[1] D. Alvis, The duality operation in the character ring of a finite Chevalley group, Bull. Amer. Math. Soc., 1 (1979), 907-911.

[2] T. Asai, On the zeta function of the varieties $X(w)$ of the split classical groups and the unitary groups, to appear.

[3] H. Azad, Semi-simple elements of ordr 3 in finite Chevalley groups, J. Algebra, 56 (1979), 481-498.

[4] C. T. Benson and C. W. Curtis, On the degrees and rationality of certain characters of finite Chevalley groups, Trans. Amer. Math. Soc., 165 (1972), 251-273, and 202 (1975), 405-406.

[5] B. Chang, The conjugate classes of Chevalley groups of type $G_{2}$, J. Algebra, 9 (1968), 190-211.

[6] B. Chang and R. Ree, The characters of $G_{2}(q)$, Instituto Nazionale di Alta Math., Symposia Math., 13 (1974), 395-413.

[7] C. W. Curtis, Truncation and duality in the character ring of a finite group of Lie type, J. Algebra, 62 (1980), 320-332.

[8] H. Enomoto, The conjugacy classes of Chevalley groups of type $G_{2}$ over finite fields of characteristic 2 or 3, J. Fac. Sci., Univ. Tokyo, Sec. I, Vol. 16, Part 3 (1970), 497-512.

[9] A. Gyoja, Liftings of irreducible characters of finite reductive groups, Osaka J. Math., 16 (1979), 1-30.

[10] N. Iwahori, Centralizers of Involutions in Finite Chevalley Groups, Lecture Notes in Mathematics, Springer-Verlag, 131 (1970), 267-295.

[11] N. Kawanaka, On the irreducible characters of the finite unitary groups, J. Math. Soc. Japan, 29, no. 3, (1977), 425-450.

[12] On the liftings of the irreducible characters of the finite classical groups, J. Fac. Sci., Univ. of Tokyo, IA, 28, no. 3 (1982), 851-861.

[13] D. Kazhdan, Proof of Springer's hypothesis, Israel J. Math., 28, no. 4 (1977), 272-286. 
[14] R. Kilmoyer, Principal series representations of finite Chevalley groups, J. Algebra, 51 (1978), 300-319.

[15] K. McGovern, Multiplicities of principal series representations of finite groups with split ( $B, N)$-pairs, J. Algebra, 77, no. 2 (1982), 419-442.

[16] T. Shintani, Two remarks on irreducible characters of finite general linear groups, J. Math. Soc. Japan, 28, no. 2, (1967), 397-414.

[17] W. A. Simpson and J. S. Frame, The character tables for $\operatorname{SL}(3, q), \operatorname{SU}\left(3, q^{2}\right)$, $\operatorname{PSL}(3, q)$ and PSU(3, $\left.q^{2}\right)$, Canad. J. Math., 25, no. 3, (1973), 486-494.

[18] T. A. Springer and R. Steinberg, Conjugacy classes, Lecture Notes in Mathematics, Springer-Verlag 131 (1970), 168-266.

[19] R. Steinberg, The representations of $\mathrm{GL}(3, q), \operatorname{GL}(4, q), \operatorname{PGL}(3, q)$ and $\operatorname{PGL}(4, q)$, Canad. J. Math., 3 (1961), 225-235.

Received June 4, 1982.

The OHio State University

Columbus, $\mathrm{OH} 43210$ 


\section{PACIFIC JOURNAL OF MATHEMATICS \\ EDITORS}

Donald BaBBITT (Managing Editor)

University of California

Los Angeles, CA 90024

Hugo RossI

University of Utah

Salt Lake City, UT 84112

C. C. Moore and Arthur Ogus

University of California

Berkeley, CA 94720
J. DugundiI

Department of Mathematics

University of Southern California

Los Angeles, CA 90089-1113

R. FINN and H. SAMELSON

Stanford University

Stanford, CA 94305

\section{ASSOCIATE EDITORS}
R. ARENS
E. F. BECKENBACH
B. H. NeUMANN
F. WOLF
K. YoshidA (1906-1982)

\section{SUPPORTING INSTITUTIONS}

\author{
UNIVERSITY OF ARIZONA \\ UNIVERSITY OF BRITISH COLUMBIA \\ CALIFORNIA INSTITUTE OF TECHNOLOGY \\ UNIVERSITY OF CALIFORNIA \\ MONTANA STATE UNIVERSITY \\ UNIVERSITY OF NEVADA, RENO \\ NEW MEXICO STATE UNIVERSITY \\ OREGON STATE UNIVERSITY
}

\author{
UNIVERSITY OF OREGON \\ UNIVERSITY OF SOUTHERN CALIFORNIA \\ STANFORD UNIVERSITY \\ UNIVERSITY OF HAWAII \\ UNIVERSITY OF TOKYO \\ UNIVERSITY OF UTAH \\ WASHINGTON STATE UNIVERSITY \\ UNIVERSITY OF WASHINGTON
}

The Supporting Institutions listed above contribute to the cost of publication of this Journal, but they are not owners or publishers and have no responsibility for its content or policies.

Mathematical papers intended for publication in the Pacific Journal of Mathematics should be in typed form or offset-reproduced (not dittoed), double spaced with large margins. Please do not use built up fractions in the text of the manuscript. However, you may use them in the displayed equations. Underline Greek letters in red, German in green, and script in blue. The first paragraph must be capable of being used separately as a synopsis of the entire paper. In particular it should contain no bibliographic references. Please propose a heading for the odd numbered pages of less than 35 characters. Manuscripts, in triplicate, may be sent to any one of the editors. Please classify according to the scheme of Math. Reviews, Index to Vol. 39. Supply name and address of author to whom proofs should be sent. All other communications should be addressed to the managing editor, or Elaine Barth, University of California, Los Angeles, California 90024.

There are page-charges associated with articles appearing in the Pacific Journal of Mathematics. These charges are expected to be paid by the author's University, Government Agency or Company. If the author or authors do not have access to such Institutional support these charges are waived. Single authors will receive 50 free reprints; joint authors will receive a total of 100 free reprints. Additional copies may be obtained at cost in multiples of 50 .

The Pacific Journal of Mathematics is issued monthly as of January 1966. Regular subscription rate: $\$ 132.00$ a year (6 Vol., 12 issues). Special rate: $\$ 66.00$ a year to individual members of supporting institutions.

Subscriptions, orders for numbers issued in the last three calendar years, and changes of address should be sent to Pacific Journal of Mathematics, P.O. Box 969, Carmel Valley, CA 93924, U.S.A. Old back numbers obtainable from Kraus Periodicals Co., Route 100, Millwood, NY 10546.

The Pacific Journal of Mathematics ISSN 0030-8730 is published monthly by the Pacific Journal of Mathematics at P.O. Box 969, Carmel Valley, CA 93924. Application to mail at Second-class postage rates is pending at Carmel Valley, California, and additional mailing offices. Postmaster: Send address changes to Pacific Journal of Mathematics, P. O. Box 969, Carmel Valley, CA 93924.

PUBLISHED BY PACIFIC JOURNAL OF MATHEMATICS, A NON-PROFIT CORPORATION

Copyright $\odot 1984$ by Pacific Journal of Mathematics 


\section{Pacific Journal of Mathematics}

\section{Vol. 113, No. $2 \quad$ April, 1984}

Alan Adolphson, On the Dwork trace formula ...................257

Amos Altshuler and Leon Steinberg, Enumeration of the quasisimplicial

3 -spheres and 4-polytopes with eight vertices .................. 269

Kenneth R. Goodearl, Cancellation of low-rank vector bundles .......... 289

Gary Fred Gruenhage, Ernest A. Michael and Yoshio Tanaka, Spaces

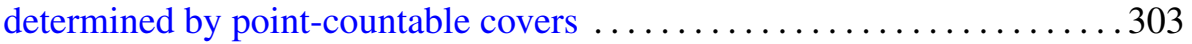

Charles Lemuel Hagopian, Atriodic homogeneous continua .......... 333

David Harbater, Ordinary and supersingular covers in characteristic $p$. . . 349

Domingo Antonio Herrero, Continuity of spectral functions and the lakes

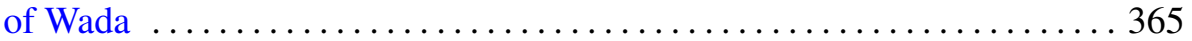

Donald William Kahn, Differentiable approximations to homotopy resolutions and framed cobordism ....................... 373

K. McGovern, On the lifting theory of finite groups of Lie type $\ldots \ldots \ldots . \ldots 383$

C. David (Carl) Minda, The modulus of a doubly connected region and the geodesic curvature-area method ............................. 395

Takuo Miwa, Complexes are spaces with a $\sigma$-almost locally finite base $\ldots . .407$

Ho Kuen Ng, Finitely presented dimension of commutative rings and modules

Roger David Nussbaum, A folk theorem in the spectral theory of

$C_{0}$-semigroups

J. S. Okon, Prime divisors, analytic spread and filtrations

Harold Raymond Parks, Regularity of solutions to elliptic isoperimetric problems

R. Sitaramachandra Rao and M. V. Subba Rao, Transformation formulae for multiple series

Daniel Ruberman, Imbedding punctured lens spaces and connected sums

Uri Srebro, Deficiencies of immersions 